\title{
Textes en francoprovençal pour l'enfance et la jeunesse
}

Textes narratifs pour introduire et motiver l'apprentissage du francoprovençal

\section{Rita Decime}

\section{(2) OpenEdition \\ Journals}

Édition électronique

URL : http://journals.openedition.org/esp/597

DOI : 10.4000/esp.597

ISSN : 2532-0319

Éditeur

Centre d'Information sur l'Éducation Bilingue et Plurilingue

Édition imprimée

Date de publication : 1 décembre 2015

Pagination : 1-12

ISSN : 1127-266X

Référence électronique

Rita Decime, "Textes en francoprovençal pour l'enfance et la jeunesse », Éducation et sociétés plurilingues [En ligne], 39 | 2015, mis en ligne le 01 octobre 2016, consulté le 14 septembre 2020. URL : http://journals.openedition.org/esp/597 


\section{TEXTES EN FRANCOPROVENÇAL POUR L'ENFANCE ET LA JEUNESSE. TEXTES NARRATIFS POUR INTRODUIRE ET MOTIVER L'APPRENTISSAGE DU FRANCOPROVENÇAL}

\section{Rita DECIME}

L'articolo (in italiano e francese) presenta una serie di esperienze condotte durante gli anni '60 e '90, in alcune classi di scuola primaria in Valle d'Aosta. Queste esperienze sono tuttora interessanti e attuali in quanto mettono in pratica $i$ principi di fondo della didattica del plurilinguismo: sfruttare le risorse linguistiche e culturali del contesto; fondare sulle conoscenze linguistiche dell'alunno in una lingua l'apprendimento di altre; favorire la messa in coerenza degli insegnamenti.

Parole chiave: Valle d'Aosta; scuola primaria; metodologia; didattica del plurilinguismo

L'article (en italien et en français) présente des expériences menées au cours des années 60 et 90, dans des classes d'école primaire en Vallée d'Aoste. Ces expériences gardent tout leur intérêt et leur actualité méthodologique car elles mettent en pratique les principes de fond de la didactique du plurilinguisme: exploiter les ressources linguistiques et culturelles du contexte; prendre appui sur ce que les élèves savent dans une langue pour en apprendre d'autres; favoriser la mise en cohérence des enseignements.

Mots-clés: Vallée d'Aoste; école primaire; méthdologie; didactique du plurilinguisme

The article (in Italian and French) presents experiments carried out during the 1960's and 90s in primary school classes of the Aosta Valley. The experiments are still interesting and up to date from the point of view of methods because they put into practice the basic principles of the didactics of multilingualism, i.e. putting all the linguistic and cultural resources of the context to use; taking advantage of what pupils know in one language in order to learn others; coordinating all the subjects to make them work together.

Key-zords: primary school; methods; didactics of multilingualism

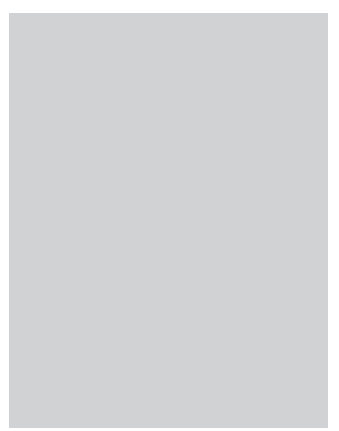

Q

uesto articolo illustra esperienze di ideazione e di costruzione di materiale didattico in francoprovenzale per le scuole del settore primario e della scuola dell'infanzia in Valle d'Aosta. Sono vicende che vanno dagli '60 agli anni '90 del secolo scorso e risentono naturalmente del clima pedagogico dell'epoca in cui sono state effettuate. Ma i principi metodologici che ne sono scaturiti vanno ben oltre questi gradi di scuola e le tecniche didattiche usate sono ritenute ancor oggi efficaci ed utili per tutti coloro che iniziano pratiche di insegnamento delle lingue, minoritarie e non.

La prima di queste esperienze risale addirittura alla fine degli 
Textes en francoprovençal pour l'enfance et la jeunesse

R. DECIME anni '60 e all'inizio degli anni ' quegli anni (siamo intorno al '68) era particolarmente vivace il dibattito sul rinnovamento della scuola, delle sue strutture e dei suoi metodi. La didattica delle varie materie, tra cui la didattica della storia, prevista dai Programmi nazionali, e di conseguenza l'Histoire de la Vallée d'Aoste, prevista dagli Adattamenti regionali, e da svolgersi in francese, erano investite da un'esigenza di rinnovamento radicale che le rendesse più vive, più critiche, maggiormente legate alle dinamiche motivazionali degli alunni e alle esigenze di una più adeguata analisi interpretativa di fatti e avvenimenti. L'avvio di questo processo di apprendimento doveva rispondere a nuove esigenze di documentazione che non fossero solo il sussidiario e basarsi su quei bisogni infantili di movimento e di azione che la ricerca psicopedagogica andava via via evidenziando.

Che significato cominciava ad assumere la parola motivazione? Si è motivati quando si è attratti da ciò che appare interessante ed è interessante ciò che risponde ad un preciso bisogno conoscitivo. Erano gli anni dell'étude du milieu che doveva motivare storia, geografia, scienze e, possibilmente, la/le lingue e l'aritmetica. Si iniziava a parlare di centri di interesse e di unità didattiche. In anni più recenti si è parlato di mappe concettuali. Sono state negli anni risposte, sempre più affinate e precise, alla necessità di ancorare sempre più le strategie dell'insegnamento alle potenzialità degli alunni e alle peculiarità del loro processo evolutivo.

Che importanza assumeva la presa di coscienza del bisogno infantile di movimento ed azione? Erano gli anni in cui si affermava il movimento dell'educazione nuova e della scuola attiva; i primi passi cioè di un percorso che avrebbe portato ad una più precisa e completa definizione dei processi di apprendimento e delle relative teorie.

In quegli anni non si parlava ancora di curricolo..., di progetto..., di offerta formativa.... L'insegnamento era ancora un'avventura individuale e solitaria dell'insegnante. Così, più semplicemente tentai, con i miei alunni l'avvio di due nuove esperienze: drammatizzare la 'grande storia', quella proposta dal sussidiario, e scrivere 'la piccola storia', quella del proprio paese, quella appena tracciata dai volumetti di histoire et géographie locale. Scopo della prima era cercare di vivificare non solo lo studio della storia, ma anche quello della lingua italiana orale e scritta; scopo della seconda era quello di motivare al meglio l'apprendimento della lingua francese andando alla scoperta delle vestigia che il territorio ancora offriva. Ambiente di quell'esperienza è stato La Thuile, piccolo paese situato ai piedi del colle del 
Textes en francoprovençal pour l'enfance et la jeunesse

R. DECIME
Piccolo San Bernardo, dove ancor oggi si possono ammirare un cromlech neolitico, due mansiones romane, la strada delle Gallie, ospizi medioevali, campi trincerati ecc., tutte vestigia che in quegli anni non erano certo valorizzate come lo sono ora.

Andare alla scoperta delle tracce che i grandi avvenimenti storici avevano lasciato nell'ambiente circostante fu una grande impresa didattica. E cercando testimonianze dirette, per forza di cose si incrociò il francoprovenzale.

Nacquero così, anno dopo anno, ispirandosi alle tecniche Freinet, giornalini scolastici in italiano e in francese. Fare le due versioni aveva lo scopo di redigere testi nelle due lingue scoprendo similitudini e divergenze: un embrione di ciò che poi i linguisti avrebbero chiamato grammatica contrastiva, che certo non ne aveva tutti i crismi, ma che rendeva quelle lezioni molto più interessanti di quelle tradizionali. Cominciava ciò̀ ad imporsi, nello studio delle lingue, una rivalutazione dei contenuti, cioè dei testi che dovevano essere significativi per gli alunni, requisito che avrebbe dovuto rendere più agevole l'apprendimento delle norme formali, cioè delle regole della grammatica e della sintassi.

Numerose persone anziane svolsero il ruolo di testimoni ed in ciò che essi raccontavano ai bambini c'era un misto di episodi storici e di leggende, di racconti veri e fantastici, di aneddoti che avevano tutto il sapore delle narrazioni atemporali tramandate di generazione in generazione. Se ben ricordo, quando si premuravano di riferire su episodi avvalorati dalla storia, e quindi anche per loro dai testi scolastici, lo facevano in italiano, qualche volta in francese. Altri testimoni invece, che per lo più riferivano episodi e vicende varie, particolarmente legati al milieu, lo facevano in francoprovenzale. Nacquero cosi altri giornalini che contenevano una storia più minuta, ma per i bambini altrettanto significativa.

Quali erano i nuovi orizzonti della didattica che queste prime esperienze lasciavano intravvedere? Quali i nuovi principi che si sarebbero progressivamente affermati? Uno di questi fu senz'altro quello di saper cogliere dall'ambiente ciò che linguisticamente può offrire e saperlo utilizzare.

Gli alunni di quell'epoca, che fossero figli di Thuileins o figli di veneti immigrati negli anni '30 per lavorare in miniera, erano per lo più patoisants; i secondi ne avevano almeno una buona competenza passiva. Italofoni unilingui erano solo i rari figli di coloro che in quegli anni erano giunti per iniziare a lavorare nelle società di sviluppo del comprensorio turistico. Il dialetto 
Textes en francoprovençal pour l'enfance et la jeunesse

R. DECIME era quindi ancora una realtà ed era una pre-competenza linguistica che andava messa a frutto. Lo intuivo e cercai di avvalermene. Se l'analisi comparativa tra le strutture dell'italiano e del francese ne metteva spesso in evidenza le divergenze, l'analisi comparativa tra le strutture del francese e del francoprovenzale poteva farne risaltare le somiglianze; e quelle strutture linguistiche i bambini le possedevano già.

Quell'esperienza confluì in una tesi di laurea sperimentale che tentò di dimostrare due dati di realtà:

- come l'insegnamento precoce del francese L2 non avesse influenze negative sull'apprendimento dell'italiano L1;

- come la padronanza del francoprovenzale costituisse una pre-competenza nei confronti dell'apprendimento del francese.

Oggi si può dire che il primo punto è assodato. La letteratura in materia è molto ampia. Il secondo è rimasto a livello di intuizione e di constatazione perché è mancato un ampio progetto di ricerca in tal senso e soprattutto il suo concretizzarsi in ben definiti strumenti didattici ad uso degli insegnanti.

La situazione linguistica dei nostri paesi, e quindi delle nostre scuole, oggi è decisamente cambiata, ma resta un principio metodologico di fondo: quello di avvalersi di ogni conoscenza, di ogni competenza che i bambini già posseggano, grande o piccola che sia, per raggiungere altri e più completi obiettivi. All'epoca sarebbe stato senz'altro più 'redditizio' ancorare l'insegnamento del francese al forte substrato francoprovenzale ancora esistente, e non partire dallo schema della lingua italiana. Così facendo si impostava, più o meno consapevolmente, l'apprendimento della Lingua 2 come traduzione della Lingua 1. I successivi studi di psicolinguistica ci avrebbero dimostrato che l'apprendimento delle lingue è un'operazione cognitivamente ben più complessa.

Altro principio efficace è quindi quello di ancorare le nuove proposte sulle competenze che gli alunni già posseggono, principio che ha per di più una grossa valenza psicologica. L'alunno si sente valorizzato ed è maggiormente recettivo alle proposte dell'adulto. Ritengo che, per quanto indebolita rispetto a quarant'anni fa, la diffusione del francoprovenzale sia ancora una risorsa da scoprire e valorizzare per impostare precocemente strategie didattiche di più ampio respiro. Le vicende riportare nell'ultimo giornalino erano una precisa espressione della comunità perché particolarmente legate a precisi individui e a determinati spazi reali. Le località evocate furono oggetto di un'esplorazione collettiva particolarmente coinvolgente. Si rese necessario quindi superare 
Textes en francoprovençal pour l'enfance et la jeunesse

R. DECIME schemi tradizionali di insegnamento che portavano a svilire una ricchezza culturale che il territorio poteva ancora offrire. D'altra parte occorreva evitare il rischio che il francoprovenzale venisse percepito come un'ulteriore materia di studio a se stante. Evidenziarne le analogie con il francese perseguiva l'obiettivo di valorizzare l'una e l'altra lingua.

Une deuxième expérience aborde plus en détail certaines de ces stratégies didactiques.

Dix ans s'étaient écoulés et je n'étais plus institutrice d'école élémentaire. Je commençais à m'occuper de l'organisation des écoles maternelles publiques. La valorisation du francoprovençal devenait à mes yeux une exigence culturelle et pédagogique toujours plus évidente. Le recueil Conte pe le petchou de inque («Contes pour les enfants d'ici») rassemble une recherche effectuée à la fin des années 70 et au début des années 80. C'était l'époque où la nouvelle école maternelle régionale commençait à construire son identité son identité d'école bilingue où, à côté de l'italien et du français, le francoprovençal devait trouver sa place et représenter un trait d'union entre les deux.

Je reprends quelques lignes de l'introduction du premier livret afin de mieux saisir le climat dans lequel on a travaillé à l'époque. Encore aujourd'hui, recréer une certaine atmosphère de recherche et de découverte rend plus aisé l'apprentissage. Dans ce cas il s'agit encore une fois de contes traditionnels, mais ces remarques peuvent également concerner d'autres aspects du milieu et s'adapter à l'âge des enfants.

"Il faut rechercher le motif principal de ce travail dans la nécessité de répondre à une double exigence:

- trouver la façon d'insérer le francoprovençal dans les activités de la classe;

- trouver le support didactique optimal sur lequel pouvoir compter.

À l'école maternelle presque toutes les activités prévoient l'emploi d'un matériel spécifique qui intègre et facilite l'action de l'éducatrice. Le problème était de découvrir le matériel le plus approprié aux activités qui se déroulent dans une langue à construire ou à perfectionner Quelles devaient être les qualités essentielles de sa spécificité?

Deux surtout nous semblèrent tout de suite évidentes. Tout d'abord, puisque le francoprovençal n'est que 'l'habit verbal' d'une culture particulière, la qualité essentielle d'un matériel favorisant son enrichissement devait être son adhérence au milieu dont il était l'expression. Ensuite il aurait fallu l'assimiler à travers un 
Textes en francoprovençal pour l'enfance et la jeunesse

R. DeCime processus d'appropriation qui renforcerait encore plus le lien entre l'enfant et son milieu.

Authenticité et possibilité d'interprétation créative devaient être les qualités requises au matériel recherché. Mais qu'est-ce que les enfants de trois, quatre ou cinq ans pouvaient rechercher et assimiler? Quelle 'opération culturelle' aurait pu les intéresser et les transformer en protagonistes?

À cette question il fut aisé de donner une réponse: les fables, le contes, les récits. Bien entendu ceux que la tradition orale rendait encore vivants et attrayants pour les petits et qui, de nos jours (à l'époque), étaient en général le patrimoine exclusif des grand-pères et des grand-mères. Tout le monde connait l'intérêt naturel que les enfants ont pour 'les histoires'. La recherche et l'exploitation de ce patrimoine oral pouvait faire d'eux de vrais protagonistes.

Toutes les institutrices utilisent des livres de fables et en font un ample usage dans la pratique didactique quotidienne. Il s'agit, dans la plupart des cas, des contes classiques de la tradition italienne et européenne. Les enfants auraient pu intégrer ce matériel à cet autre qui, encore inédit et sans doute plus modeste, les touchaient indubitablement de plus près. Dans cette entreprise, parents, grands-parents, amis, 'experts de village' ont été engagés.

Il existe déjà dans le commerce des recueils de récits et légendes de la Vallée d'Aoste, mais souvent ils présentent un double inconvénient: ils ne sont pas toujours à la portée de la compréhension d'enfants si petits, mais surtout rarement ils sont écrits en francoprovençal et chacun sait qu'une traduction est une tout autre chose.

Se servir de ce qu'on connaît déjà pour l'adapter peut être une opération plus simple, mais d'une signification différente. L'enfant est toujours charmé par l'histoire du Chat botté ou de Cendrillon: le fantastique le séduit instinctivement. Mais il dispose de ces récits dans l'une ou l'autre des langues de l'école (italien et français). Dans son dialecte il aurait reconstruit quelque chose de beaucoup plus simple, de bien moins fantastique et pourtant de très significatif.

Ces livrets de Contes pe le petchou de inque ont privilégié les histoires qui avaient comme protagonistes les animaux. Ces histoires étaient d'ailleurs les plus nombreuses et c'est justement la simplicité des aventures qu'elles racontaient qui s'adaptait le mieux à la capacité de compréhension et d'intériorisation des petits. Certaines ont été reprises telles quelles de la réalité et transmise 
Textes en francoprovençal pour l'enfance et la jeunesse

R. DeCIME

\section{QUELQUES RÉFLEXIONS ENCORE SUR LA MÉTHODOLOGIE ADOPTÉE}

à cause de leur caractère exceptionnel. D'autres sont peut-être la synthèse d'évènements divers mis ensemble par la fantaisie et la personnalité du narrateur. D'autres encore ont sans doute été enrichies par des images inédites. Mais leur charme est dû aussi à tout ce qui peut être ajouté et rajouté chaque fois par celui qui raconte. Dans le monde de la télévision la tradition orale du conte est en train de se perdre et avec elle la capacité de se souvenir, de recoudre les événements, de créer des images mentales; activités fondamentales pour l'apprentissage des langues, et non seulement."

Dès le début, les contes étaient présentés en patois, par l'institutrice ou par l'intermédiaire de chaque narrateur. C'était en effet la condition indispensable pour se servir dès le début de la langue du village, avec laquelle définir les lieux, les personnages, les évènements qui auraient ainsi conservé leur 'dimension verbale' même dans les expériences didactiques suivantes.

Dans certaines classes on a commencé par un récit trouvé par l'institutrice. Dans d'autres, plus favorisées, on a trouvé dès le début des narrateurs enthousiastes, heureux qu'un petit public se forme autour d'eux. Il est certain que le fait d'aller tous ensemble écouter une histoire prenait immédiatement l'aspect d'une découverte collective et ceci facilitait toutes les exploitations successives. Au moment de la reconstruction en classe, il n'y avait pas seulement une version à interpréter, il y avait plutôt de nombreux souvenirs à faire revivre et à rassembler. L'histoire était reconstruite, réinterprétée et revécue avec la participation de tous.

Le contenu de chaque histoire a motivé aussi des expériences didactiques différentes. Au-delà des activités de verbalisation, le francoprovençal a été employé pour des activités de peinture, de modelage, de construction, de dramatisation, de reconstruction ludique. Chaque histoire a constitué une unité didactique plus ou moins ample qui a déclenché chez les enfants des fonctions sensorielles, perceptives, psychomotrices, représentatives, symboliques, cognitives. Le francoprovençal avait su recouvrir tous les secteurs d'expérience qu'on avait développés. Les réinar (les renards) et les dzeleunne (les poules), les verdzasse (les écureuils) et les pavioule (les papillons) étaient restés tels quels, dans leur spécificité linguistique, du jour de la première narration à celui du dernier jeu libre. La langue qui avait marqué leurs découvertes avait pu accompagner aussi leurs expériences successives.

Pour les petits chez lesquels la compétence en patois était insuffisante, l'institutrice devait d'abord essayer de mettre en valeur le 
Textes en francoprovençal pour l'enfance et la jeunesse

R. DECIME bagage lexical déjà acquis, de le diriger vers un usage finalisé, de faciliter la compréhension intuitive des expressions encore inconnues et de favoriser la production verbale en situation.

La réception des contes à travers la télévision habitue les enfants à un plaisir individuel; en général ils font cette expérience seuls, isolés les uns des autres. Chacun ne peut compter que sur sa propre capacité de réception. Au contraire, écouter une histoire avec des camarades favorise la socialisation et aide à surmonter l'égocentrisme typique de cet âge, car les sensations, les émotions, et les sentiments les plus divers sont partagés. L'enseignant peut parler, répéter, enrichir, exploiter chaque intervention en donnant des explications; il peut susciter des réactions, proposer des images qui facilitent chez l'enfant le processus d'identification entre les aventures des différents personnages et les siennes. Mais pour que ceci puisse se produire, la communication doit être 'biunivoque': l'enfant doit pouvoir intérioriser et extérioriser, il doit pouvoir prendre part au récit, se sentir l'interprète et le collaborateur d'une activité collective.

Le langage de l'enfant s'articule en une succession de phases que sa croissance intellectuelle conditionne et dont elle est le reflet. À l'âge de l'école enfantine et de l'école primaire l'élève mobilise toutes ses ressources pour conquérir la façon de parler des adultes qui l'entourent. Il faut faire en sorte que ce langage ne soit ni réduit ni stéréotypé, ni privé de nuances qualitatives et adverbiales comme l'est souvent le modèle linguistique que la télévision propose. La revalorisation des parlers dialectaux est aussi, dans le fond, la réaffirmation du droit à la créativité linguistique.

Dans la pédagogie ses années 1980-90 le terme Unité didactique, était d'extrême actualité et s'inspirait de la méthodologie des Centres d'intérêt conçue par O. Décroly. Un besoin important était ressenti; celui de dépasser les stratégies un peu simplistes des méthodes audio-visuelles, telles que Frère Jacques, Bonjour Line et autres, méthodes basées sur une programmation stricte de l'apprentissage des structures linguistiques et sur les exercices de dénomination et de répétition qui devaient avoir pour but d'éviter l'effet néfaste des erreurs. On avait compris que le processus d'acquisition d'une nouvelle langue devait se réaliser toujours progressivement, mais à travers différents types d'expérience qui reproposait certaines structures linguistiques à des moments différents de communication ou d'explicitation de la pensée.

Un conte, une légende, un récit devenaient alors un prétexte pour susciter l'intérêt des enfants, mais les structures linguisti- 
Textes en francoprovençal pour l'enfance et la jeunesse

R. DECIME ques que l'on voulait faire apprendre devaient réapparaitre dans des moments didactiques les plus diversifiés possibles.

Pour apprendre une langue orale ce n'est pas indispensable de définir a priori un ordre de présentation des différentes constructions grammaticales; il vaut beaucoup mieux enrichir le plus possible l'expérience de l'enfant en lui proposant un répertoire d'activités organisées sur l'univers évoqué par le texte. Faire réapparaitre des mots, des phrases et des structures dans un contexte d'expériences le plus vaste possible permet à l'enfant d'appréhender sous des formes diverses les significations proposées dans le texte de départ et de faire fonctionner le vocabulaire dans des situations réelles de communication.

La diversité des expériences assure à chacun des voies d'accès au sens qui lui sont propres.

Il faut être conscients qu'un mot est un signifiant qui ne peut être utilisé pour exprimer un signifié que dans la mesure où celui-ci existe pour l'enfant, c'est-à-dire qu'il a déjà été perçu et organisé par lui à un niveau non-verbal. Ce qu'un mot exprime c'est un ensemble de significations élaborées par l'élève au cours d'expériences vécues dans le courant d'activités motrices, perceptives ou cognitives. Toutes ces activités, adaptées bien sûr à l'âge des enfants, permettent de réutiliser et de faire varier le vocabulaire, ainsi que les constructions syntaxiques, dans des situations sémantiquement proches; elles contribuent à enrichir le matériel linguistique offert à l'apprenant et favorisent une première apparition de productions autonomes.

Il faut savoir distinguer la répétition d'un énoncé mémorisé de la production autonome faite par un sujet qui a intériorisé les règles qui lui permettent de construire un énoncé.

Ce n'est que par un processus très lent que le sens complet des mots se met en place. Il faut donc donner une importance toute particulière aux tentatives que fait l'apprenant pour exprimer sa pensée, même si au début elles débouchent sur des erreurs, et à toute situation qui peut faciliter la verbalisation.

Con questa metodologia di lavoro è stato raccolto, organizzato e utilizzato didatticamente anche un materiale leggendario e favolistico relativo a «Racconti, fiabe e leggende della Valle d'Ayas». La ricerca è coeva a quella de le Conte pe le pechou de inque, ma la stesura e la pubblicazione del materiale è molto più recente e ha dato luogo ad un materiale didattico, o di semplice lettura, che si adatta anche ad alunni più grandi di quelli del settore primario. I testi contenuti nel volume sono quelli che ancora si raccontavano nelle veillà prima dell'arrivo della televisione. 
Textes en francoprovençal pour l'enfance et la jeunesse

R. DECIME
Non è stata fatta una selezione che privilegiasse questo o quel tipo di racconto. I testi sono di vario genere. L'articolazione nei seguenti capitoli ha il semplice scopo di riordinare, secondo temi diversi, l'ampio materiale raccolto.

-Luoghi che custodiscono dei segreti,

-Uomini e animali,

- Anime che ritornano dal paese dei morti,

- Tra verità e leggenda,

- I racconti degli zoccolai

- Per i più piccoli.

Si può leggere nell'introduzione:

"Trascrivere queste storie è stato un po' come narrarle nuovamente ad un pubblico immaginario, è stato un tentativo di ridare loro una nuova vita, rilanciarle come messaggi che, travalicando le scadenze temporali, possano contribuire a far nascere, o a consolidare, un sentimento di condivisione e quindi di appartenenza. Trascriverle è stato anche affidarsi alla speranza (o all'illusione) che il tempo del raccontare non sia ancora del tutto passato, ma che possa riemergere, magari in forme nuove e più attuali".

Di tanti racconti è stata data la versione in patois, che corrisponde esattamente a quanto è stato registrato dalla viva voce dei narratori. Si tratta di testi caratterizzati da una particolare semplicità strutturale, le cui enunciazioni linguistiche, concise, leggere, essenziali, sembrano pensate e dette in economia. Tutti hanno una più ampia versione in italiano, che si impone per ovvi motivi di fruizione e di diffusione, e una versione in francese. Quest'ultima è una traduzione, la più letterale possibile, delle storie in patois e un riassunto di quelle che hanno solo la versione italiana; il suo scopo è quello di facilitare al massimo la comprensione, nonché l'analisi linguistica mediante confronti e approfondimenti. Ovviamente si sono incontrate difficoltà di traduzione da una lingua all'altra. Non sempre i concetti che un codice esprime sono perfettamente trasferibili in un codice diverso, anche se affine. Inoltre non sempre si riescono a riprodurre in un'opera scritta tutte le sfumature espressive di un testo originariamente orale".

Quanto è già stato detto per le esperienze precedenti è valido anche per quest'ultima. Una scuola aperta al plurilinguismo deve innanzitutto saper far tesoro della ricchezza linguistica del proprio territorio.

Cette digression historico-pédagogique nous amène aux années 2000. La recherche psycholinguistique et didactique nous a pro- 
Textes en francoprovençal pour l'enfance et la jeunesse

R. DECIME posé des concepts nouveaux et, parmi ceux-ci il y avait, et il y a toujours, la notion de curriculum. Les définitions qui le concernent étaient et sont encore multiples, mais, dans la réalité, il proposait et il propose de mettre en harmonie trois aspects de l'action éducative:

- ce que l'enseignant doit faire (selon les programmes ou le projet de l'école),

- ce qu'il pense pouvoir faire dans sa classe (selon sa composition),

- ce que l'élève fait effectivement (c'est-à-dire son parcours d'apprentissage).

Ce dernier point, qui concerne plus particulièrement l'élève, est le plus essentiel.

Si le curriculum, entendu comme parcours d'apprentissage, repose sur la conscience de ce qu'il fait et de la manière dont il le fait, cela suppose non seulement une explicitation claire de la part de l'enseignant de ce qu'il va lui proposer, mais cela suppose aussi et surtout une forte prise en compte et une valorisation de ses apports

Expliciter et permettre la prise de conscience des raisons et des buts d'une action veut dire assumer une attitude méthodologique de fond qui facilite l'activité de représentation mentale de l'élève. Il faut être particulièrement sensibles à ce travail de représentation et faciliter une élaboration langagière constante des expériences conduites.

Enseignant et élève doivent prendre conscience de façon explicite de la liaison essentielle, de l'unité et de la cohérence des activités proposées.

C'est la condition indispensable pour que l'apprenant puisse transformer la succession des différentes activités en l'histoire personnelle de ses apprentissages.

Unité et cohérence renvoient à une idée d'interactivité forte entre celui qui enseigne et celui qui apprend. Ce qui change c'est la nature du travail demandé à l'élève: on n'est absolument pas dans un modèle question/réponse ou de consigne à exécuter. L'organisation même d'une situation d'apprentissage doit permettre aux apprenants de construire le sens de ce qu'ils entendront et de ce qu'ils feront.

C'est dans l'attention à l'activité cognitive de l'élève que ce qu'on lui propose comme expérience peut être porteur d'unité et de cohérence et activité cognitive signifie comprendre, réfléchir, échanger, généraliser formuler, c'est-à-dire conceptualiser.

Chaque individu développe automatiquement cette activité mentale en langue maternelle. Les représentations mentales sont toujours des représentations langagières qui concernent la lan- 
Textes en francoprovençal pour l'enfance et la jeunesse

R. DeCIME gue intérieure dont chacun dispose. On ne passe pas spontanément à une langue seconde ou troisième: il faut donc laisser travailler les élèves avec les outils langagiers qui leur sont propres, accepter au début la micro-alternance et pousser la nouvelle langue quand les contenus de l'expérience proposée sont bien représentés. Il faut accepter au début de passer à travers une langue qui est approximative, en cours de construction et faire crédit à l'enfant qu'il est en train d'élaborer des connaissances nouvelles et qu'il le fait avec des mots nouveaux.

Un accent particulier peut être mis sur le tutorat des élèves, les uns par rapport aux autres. Leurs connaissances de départ ne sont pas toujours du même niveau. L'effort de l'enseignant est de faire en sorte qu'ils se sentent compétents et puissent mettre leur compétence à la disposition des autres. En cours d'activité il doit les rendre conscients de leurs apprentissages, de leurs progrès, de leurs compétences. Il doit les inviter à les utiliser pour aider les autres et aussi à les expliciter pour en faire des outils transférables dans d'autres situations.

On peut facilement comprendre quelle est l'importance des expériences qu'on propose. L'enseignement/apprentissage d'une langue n'est pas une affaire simple et se joue beaucoup sur le type de conduite pédagogique qu'on adopte et qu'on poursuit. 